éléments d'une méthode générale de recherche et de numération du B. Coli dans le lait dans tous les cas possibles. Cette méthode a été exposée en détail dans le présent article.

Nous croyons qu'elle pourra, par sa simplicité et sa rigueur, rendre quelques services dans l'étude bactériologique des laits et principalement des laits très purs et des laits sortant des pasteurisateurs industriels.

\title{
RECHERCHES SUR LA CASÉINE
}

\author{
par Mlle J. BRIGANDO \\ Docteur ès Sciences de $1^{\prime}$ Université de Lyon, \\ Chef de Travaux à l'Institut des Recherches Agronomiques.
}

(Suite.)

\section{PRÉPARATION DE LA CASÉINE-AGIDE DANS L'INDUSTRIE}

Nous allons maintenant nous occuper de la préparation de la caséine-acide industrielle. Toutefois nous tenons à rappeler comment on la prépare au laboratoire, afin de puiser dans la technique suivie les raisons d'une amélioration de la fahrication industrielle.

L'irrégularité de celle-ci est un fait bien connu sur le terrain commercial. La caséine est un sous-produit auquel on pense quand il y a un surplus de lait écrémé qui ne peut pas être utilisé dans l'alimentation humaine ou celle des animaux. La fabrication de la caséine-acide peut cependant être améliorée comme nous nous efforcerons de le montrer au cours de ce travail.

Dans les pages antérieures, nous nous sommes employée à marquer la différence profonde qui sépare la caséine-acide de la caséine-présure. Nous avons vu que l'acidification du lait en vue de l'obtention de la caséine-acide peut être réalisée de diverses façons; nous pouvons laisser le lait s'acidifier spontanément ou bien ajouter un acide, en proportion donnée, et dans des conditions déterminées.

Dans ce chapitre, et afin d'éviter toute confusion, nous appellerons caséine-lactique, la caséine d'acidification spontanée, et caséineacide, celle qui résulte de l'acidification provoquée par l'apport d'un acide minéral ou organique autre que l'acide lactique. Cette distinction est d'ordre industriel et elle est faite pour éviter des confusions de langage, car, théoriquement, on ne voit pas sur quoi elle peut se baser; on pourrait très bien se servir d'acide lactique industriel pour précipiter la caséine du lait frais. On effectuerait ici l'apport d'un acide qui, habituellement, est préparé par les ferments lactiques, commensaux habituels du lait.

Les divers acides employés. - Les acides employés lors de 
l'acidification provoquée sont des acides minéraux et, quelquefois, organiques : acide chlorhydrique, acide sulfurique, acide acétique. Théoriquement, la nature de l'anion uni à l'ion $\mathbf{H}$ dans l'acide employé, importe assez peu. Le choix de l'acide paraît donc indifférent. Cependant, dans la pratique, il est assez limité, ainsi qu'on en peut juger par la liste très courte des acides que l'industrie emploie et que nous venons de donner.

Ce choix estfixé surtout par des considérations, chimiques, d'une part, économiques et physiques d'autre part. L'acide azotique ne saurait être employé, moins peut-être parce que c'est un acide oxydant que parce qu'il colore les matières protéiques. Sans doute, sa dilution est grande, mais on chauffe et il y aurait toujours à craindre l'action de l'acide sur les substances azotées.

Si le sérum est employé pour la fabrication du lactose, c'est à l'acide chlorhydrique qu'il faut penser, et non pas à l'acide sulfurique, le sulfate de calcium qui se forme pouvant gêner l'obtention du sucre de lait. L'acidification spontanée, la self-acidification, n'est également pas à recommander si l'on veut retirer du sérum le sucre de lait. On est gêné, dans la cristallisation du lactose, par les lactates formés en abondance. Il faut toujours ici recourir à l'acidification provoquée. L'acide phosphorique ne peut pas être employé comme précipitant de la caséine, du fait de son prix élevé, même si l'on utilisait l'acide commercial.

Les considérations physiques que nous pouvons faire valoir dans le choix d'un acide, lors de l'acidification provoquée du lait, doivent tenir surtout à la valeur de la constante de dissociation de l'acide. Un acide fort étant plus dissocié qu'un acide faible, il en faut une moindre quantité pour obtenir le même résultat.

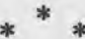

\section{LES TEMPS ESSENTIELS DE LA FABRICATION.}

Essentiellement, la fabrication de la caséine-acide se ramène à :

a) précipiter la caséine ;

b) la laver ;

c) la presser;

d) la moudre ;

e) la sécher.

Voyons d'abord les procédés de préparation au laboratoire pour en retenir, avons-nous dit, ce qui est d'application à la préparation industrielle. Nous trouvons, dans la thèse de $\mathrm{Ch}$. Porcher, le détail des techniques de Hammarsten et de L. L. van SLyke et J. C. Baker. 
La technique de O. Hammarsten.- O. Hammarsten étend le lait de quatre volumes d'eau distillée et l'additionne d'une quantité suffisante d'acide acé tique dilué, pour que le taux de celui-ci atteigne, dans la liqueur, 0, 075-0,100\%. La caséine se dépose très vite et après avoir siphonnél'eau, on la redissout dans la plus petite quantité possible d'ammoniaque et on lạ reprécipite. On recommence deux à trois fois les précipitations et les redissolutions et, la dernière fois, la caséine est lavée jusqu'à ce que toute trace d'acide soit enlevée. On filtre. La caséine recueillie est légèrement pressée, puis lavée à l'alcool fort, ensuite traitée par l'éther, une fois qu'on l'a débarrassée de l'alcool.

"L'importance du point isoélectrique, dit Ch. PORCHER, nous permet de régler aujourd'hui, avec beaucoup de précision, la précipitation de la caséine, de façon à la réaliser exactement à ce point caractérisé par un $p \mathrm{H}=4,6-4,7$, correspondant à une concentration en ions $\mathrm{H}=2,5 \times 10^{-5}$.

"Réserve faite de cette observation, la technique de ceux qui préparent de la easéine au laboratoire suit, dans ses lignes essentielles, celle qui a été indiquée en premier lieu par 0. Hammarstan. L'observation d'une acidité stricte lors de la précipitation, d'une. alcalinité non moins stricte lors de la redissolution, est done facilitée aujourd'hui par notre connaissance du $p \mathrm{H}$ et des moyens de l'obtenir d'une façon précise. "

La technique de L. L. Van Slyke et J. C. Baker. - Cette technique (23) n'est qu'une amélioration de celle qui avait été exposée quelques années auparavant par L. L. VAN SLYKE et A. W. Bosworth.

L'idée qui a guidé L. L. VAN SLYKE et J. C. BAKER, c'est de se rapprocher, par une acidification provoquée, de ce qui est réalisé spontanément par la fermentation lactique régulière du lait. Lear technique conduit, disent ces auteurs, à une caséine pure, plus pure que celle qui est obtenue par le procédé de Hammarsten. Elle se ramène à ceci : addition graduelle d'acide dans la masse du lait refroidi à $15^{\circ}$, addition très lente et surveillée pendant que le liquide est remué violemment par un agitateur à palettes faisant 2.000 à 3.000 tours par minute. Il faut veiller à ce que la température ne s'élève point. L'acide dilué est porté au fond du liquide par un tube capillaire à extrémité effilée.

L. L. VAN SLYKe et J. C. BAKER notent que l'acide chlorhydrique, seul, donne des résultats excellents pour la précipitation. Ces auteurs surveillent avec le plus grand soin, en s'aidant d'indicateurs sensibles, tel que le pourpre de bromo-crésol, pour déceler le moment où cette précipitation approche.

Lorsqu'on dilue du lait acidifié dont le $p H$ n'est pas loin du point

(23) L. L. VAN SLYKE et J. C. BAKER. Studies relating to milk. The preparation of pure casein. New-York Agric. Exp. Stat. Tech. Bull., décembre 1918, nº 65. 
isoélectrique de la caséine, la séparation de la caséine se fait brusquement, parce que l'addition d'eau distillée accentue l'ionisation de l'acide ajouté et augmente, par cela même, la concentration en ions $\mathrm{H}$ du lait acidifié, si bien que la dilution permet d'atteindre ainsi le point isoélectrique tout près duquel on était arrivé par l'apport lent de l'acide.

Cebte observation montre toute la sensibilité du procédé employé.

Relevons ce point important dans la technique de L. L. vaN SLYKE et J. C. BAKER, c'est qu'il n'y a pas de dilution préalable du lait, comme dans le procédé de Hammarsten ou dans celui que Ch. Porcher indique dans sa thèse et que nous avons appliqué à son laboratoire. Il n'y a pas non plus, dans le procédé de L. L. VAN SLYKE et J. C. BAKER, de redissolutions ultérieures avec reprécipitations.

L'essentiel du premier temps de la précipitation de la caséine, selon la technique de L. L. VAN SLYKE et J. C. BAKER se ramène done à ceci :

$1^{\circ}$ On précipite à basse température (pas plus de $1.5^{\circ}$ );

$2^{\circ}$ L'apport d'acide est extrêmement lent ;

$3^{\circ}$ La répartition de l'acide est très régulière, du fait de cette lenteur, d'abord, et aussi du fait que le liquide est agité très vigoureusement, ce qui permet le renouvellement des surfaces de contact entre le lait qui va être attaqué et l'acide qui attaque.

Nous allons maintenant faire suivre l'examen de la préparation au laboratoire, de l'exposé des diverses techniques industrielles.

- Nous empruntons notre documentation, en ce qui concerne la fabrication française, à l'ouvrage de P. DoRnic et A. ChOLLET, et, en ce qui concerne la fabrication américaine, à O. DAHLBERG ainsi qu'à des documents argentins personnels qui ont été offerts au Professeur Porcher, lors de ses voyages dans ce pays.

\section{LES TECHNIQUES FRANÇAISES.}

caséine lactique. acidification spontanée (d'après P. Dornic et A. GHoLLes.) - L'hiver, on active la fermentation naturelle, soit en réchauffant le lait quand la cuve est pleine, soit en ajoutant au lait écrémé une certaine quantité de levain préparé avec des ferments lactiques purs, dans les mêmes conditions que pour l'ensemsncement des crèmes pasteurisées. On peut enfin se contenter de garder un pэu de lait caillé de la veille, avant la cuisson de la caséine, pour mottre dans la préparation du lendemain. Le plus souvent, l'addition de 2 à $4 \%$ de levain suffit.

"Pour avoir un rendemænt aussi élevé que possible, il faut que la caséine soit nettement séparée du sérum et forme ce que les ouvriers appellent « le chapeau », 
A ce moment, on brise le caillé, généralement avec une pelle en bois. Le caillé est ensuite cuit, comme dans la fabrication de la caséine à la présure (a).

CASÉINE-ACIDE D'ACIDIFICATION PROVOQUEE. - " La fabrication de cette sorte de caséine est très réduite en France, nous disent P. Dornic et A. Chollet. Seuls, sont utilisés l'acide sulfurique et l'acide chlorhydrique (1)

“Voici le mode opératoire suivi à la laiterie coopérative de Sains-du-Nord et qui est dî à M. Courthéoux, l'administrateurdélégué :

"On dilue l'acide sulfurique du commerce à $66^{\circ}$ Baumé dans 4 à 5 volumes d'eau, en ayant le soin habituel de verser l'acide dans l'eau en agitant continuellement. Si l'on veut que le sérum serve à l'alimentation des pores ou si la caséine devait être utilisée dans l'alimentation humaine, il faut prendre de l'acide sulfurique pur, exempt d'arsenic.

“Le lait écrémé est chauffé vers $45^{\circ}$ et, en agitant, on verse l'acide. La précipitation se fait rapidement; on poursuit l'agitation pour obtenir des grains de caillé suffisamment fins. On continue à chauffer, toujours en agitant, jusqu'aux environs de $70^{\circ}$. La caséine est ensuite lavée, pressée et séchée, comme les autres caséines. $p$

\section{LES TECHNIQUES ARGENTINES.}

CASÉINE LACTIQUE D'ACIDIFICATION SPONTANÉE, - La technique qui suit ressemble beaucoup à la technique française. Nous la donnons néanmoins avec les détails qui ont été recueillis sur place.

Le chauffage du lait avant le passage à l'écrémeuse s'opère, le plus souvent, en introduisant directement le tuyau de vapeur dans les cuves en bois ; il est recommandé d'effectuer la précipitation dans des cuves à double fond en cuivre, comme e'est le cas dans certaines laiteries. Ces cuves ont environ $2 \mathrm{~m} .40$ de diamètre et $1 \mathrm{~m} .20$ de hauteur. Le lait écrémé se rend directement dans les cuves, au sortir de l'écrémeuse, et s'il est nécessaire d'utiliser une pompe, il est préférable de faire usage d'une pompe à piston et non pas d'une pompe centrifuge qui provoque une formation excessive de mousse. L'écrémage doit se faire à une température de $37^{\circ}-38^{\circ} \mathrm{C}$. et, s'il a lieu à $52^{\circ}$, commo certains le recommandent, on obtient de mauvais résultats. Ensuite, le lait est laissé dans les cuves au repos jusqu'à ce qu'il soit caillé entièrement dans sa masse. Selon l'acidité originelle du lait et la saison, la coagulation peut demander de 10 à 24 heures. Une fois le lait uniformément caillé, on le chauffe et l'on porte sa température jusqu'à $50^{\circ}$ sans brasser. On brasse énergiquement ensuite, en élevant progressivement la température jusqu'à $60-62^{\circ}$, afin de réduire le caillé en grains, de la grosseur approximative d'un petit grain de maîs. Si l'on commence le brassage plus tôt, avant que le lait n'ait été chauffé jusqu'à $50^{\circ}$, on risque de réduire le caillé en grains trop petits, et le rendement industriel est plus faible. Une caséine un peu poussiéreuse serait obtenue, qui s'en irait par la bonde, avec le sérum, lors de l'évacuation de ce dernier.

La cuisson a pour but de durcir les grains de caséine qui se rétractent. On

(a) Voir plus loin, pour la cuisson de la caséine-présure.

(1) Il faut ajouter à ces acides l'acide acétique employé dans une laiterie du Sud-Ouest. 
arrête l'opération lorsque, en serrant la easéine dans la main, elle donne une masse compacte dont les grains restent séparés les uns des autres, en laissant exsuder un sérum verdâtre et transparent. On sépare la caséine précipitée du sérum, et on lave soigneusement à l'eau froide.

\section{CASÉINE D'ACIDIFICATION PROVOQUEEE PAR DES SERUMS LACTI-}

QUEs. - Une seconde technique est très répandue en Argentine, ainsi que le Professeur Porcher a pu s'en rendre compte de visu. Ici, la préparation de la caséine-acide se fait par un procédé analogue à celui que nous allons examiner tout à l'heure, lorsque nous parlerons des techniques américaines.

Ce procédé consiste à additionner au lait écrémé et chauffé une quantité de sérum acide d'opérations antérieures. Les baes de ces sérums-acides sont souvent envahis de fermentations anaérobies secondaires, et il s'en échappe une odeur désagréable.

Los sérums employés dans cette technique sont parfois extrêmement vieux. On ajoute tous les jours à la même cuve un peu de sérum acide de l'opération qui a été effectuée le jour même. On force toujours la dose du sérum et l'on a des caséines extrémement acides. Toute la masse est chauffée aux environs de $40^{\circ}$ à $42^{\circ} \mathrm{C}$., et cette température est trop faible pour que le grain de caséine précipite au fond de la euve; il remonte à la surface où il forme une masse spongieuse que l'on pout estimor être un mélange de caséine précipitée, avec la mousse du petit-lait, notamment lorsque le pompage du lait a été effectué par des pompes centrituges. Le lavage d'une telle masse est difficile.

En général, on ne presse pas suffisamment la caséine avant de la passer au " moulin à caillé "; elle renferme une forte proportion de sérum. On l'étend sur des claies, et on la fait sécher au soleil. La caillebotte étant très humide, le séchage est lent ; il y a des fermentations anaérobies ; la caséine jaunit et prend une odeur nauséabonde.

On fait, en général, le séchage au soleil, dans des terrains qui ne sont pas abrités contre le vant. Lo vent, surtout par temps sec, soulève des poussières qui salissent la caséine et la randent grisâtre. Aussi, la caséine obtenue, déjà fort acide, renfermet-elle beaucoup de points noirs.

\section{LES TECHNIQUES AMÉRICAINES.}

Nous n'insisterons pas sur la forme des cuves qui, en Amérique, sont en général rectangulaires et semblables aux cuves à fromages de Cheddar. Elles sont pourvues à une extrémité de valves grillagées pour l'écoulement du sérum. Leur contenance va de 4 à 6.000 livres de lait écrémé, soit 1.800 à 3.000 litres. De plus en plus, on les munit d'un agitateur mécanique, mais, dans beaucoup de cas, elles n'en possèdent point et l'agitation du lait, notamment lorsqu'il s'agit d'acidification provoquée, est extrêmement rudimentaire.

Comme le fait remarquer A. O. DahlBerg (24), le mode de fabrication est, en général, à peu près toujours le même ; mais nous verrons combien retentit sur la minéralisation de la caséine et, par-

(24) O. Dahlberg in Casein and its industrial applications de E. SUtermetster. 
tant, sur ses propriétés commerciales, la façon de procéder à la. précipitation de la caséine.

PROcÉde d'Acidification NATURELle. - La caséine lactique obtenue par acidification naturelle est le type de caséine que le commerce regarde comme ayant habituellement la meilleure qualité pour les usages généraux, en raison de sa meilleure solubilité et de sa plus faible viscosité quand on la dissout.

Le lait écrémé, au sortir de l'écrémeuse, est conduit dans des cuves en bois où on le garde à une températura favorisant le développement de l'acide lactique.

Quand le processus d'acidification est sur le point d'être terminé, on ajoute à la masse, aussitôt que possible, 5 à $10 \%$ d'un sérum acide antérieur, pour hâter la fermentation. Une partie du sérum obtenu des opérations précédentes est gardée de jour en jour à une température qui va de $32^{\circ}$ à $43^{\circ}$, comme source de ferments lactiques pour l'ensemencement.

Quand le lait écrémé a une acidité suffisante pour donner un caillé mou, on le ehauffe directement à la vapeur, pendant qu'on agite vigoureusement avec un râteau en bois, jusqu'à ce qu'on obtienne une séparation nette du caillé et du sérum. Une température de $43^{\circ}$ à $52^{\circ} \mathrm{C}$. $\left(110^{\circ}\right.$ à $125^{\circ} \mathrm{F}$.) donne les résultats désirés. L9 caillé se ramasse au fond de la cuve en une masse plus ou moins compacre, laissant le sérum clair. Ce sérum est soutiré au travers d'un sae attaché à la valve grillagée pour retenir les toutes petites particules de caillé qui, autrement, seraient perdues. Comme nous l'avon's vu plus haut, une partie de ce sérum êst conservé pour le lendemain.

Le caillé est lavé en le couvrant avee de l'eau froide et en agitant bien. Après qu'on a drainé l'eau de lavage, le caillé est prêt pour être pressé.

La trop grande acidité du lait écrémé, au moment du chauffage, donne un caillé plus fin, plus dur, plus difficile à manier sans l'emploi d'un tamis d'égonttage, tandis qu'une acidité insuffisante empêche une séparation nette et complète du caillé. C'est l'expérience qui indiquera à l'opérateur, dit A. O. DAHLBERG, l'acidité la plus convenable et les conditions optima pour obtenir les meilleurs rendements.

Avec un bon levain (un "starter ", comme on dit aux Etats-Unis), il faut 10 à 15 heures au lait écrémé sortant chaud, - aux environs de $35^{\circ}$, - de l'écrémeuse, pour acquérir une acidité suffisante, laquelle donnera un bon travail lors de la préparation de la caséine.

Si le lait écrémé doit rester plus longtemps, comme cela s'observe quand on travaille sur du lait reçu le soir, il faut régler la température de la cuve et la quantité de levain, afin que le lait ne caille pas trop longtemps avant le chauffage. Celui-ci doit être réalisé le plus rapidement possible après que le lait écrémé a coagulé avec la eonsistance désirable, afin d'obtenir un caillé qui puisse être manipulé facilement. 
Si le lait écrémé caillé tarde à être travaillé, il faut le chauffer à une température plus élevée pour obtenir un rendement satisfaisant, mais cette température ne devra jamais excéder $57^{\circ}$ à $60^{\circ}$.

METHOdE A L'ÉJECTEUR. - Si on laisse s'écouler du lait écrémé bien acidifié, présentant un caillé ferme, mais néanmoins diffluent, d'une cuve dans une autre cuve inférieure, en le faisant passer en face d'un éjecteur de vapeur, on obtient une rapide séparation d'un caillé granuleux très favorable au lavage.

Le lait écrémé frais est pompé dans une cuve élevée, et on le laisse s'acidifier jusqu'à ce qu'un caillé se soit développé. Du sérum acide, d'opérations antérieures, peut être employé pour faciliter le développement de l'acidité, mais, le plus souvent, on laisse le lait écrémé s'acidifier complètement.

Si le chauffage avec l'éjecteur a été effectué avant que le lait ne montre un coagulum ferme, on obtient un caillé souple, caoutchouteux, impossible à travailler convenablement. C'est dire que, si l'on manque de temps pour obtenir une bonne coagulation du lait écrémé, il vaut mieux recourir à la méthode précédente de l'auto-acidification en cuve.

La cuve dans laquelle s'effectue l'auto-acidification doit être suffisamment élevée pour permettre au caillé du lait écrémé de s'écouler, de passer d'abord devant l'éjecteur, ensuite dans la cuve inférieure dont le fond est constitué par un tamis qui retient la caséine. Ce dispositif de travail permet d'utiliser le minimum de yapeur nécessaire. Dэ plus, il exige peu de main d'cuvre et de force.

Les dimensions et le nombre des éjecteurs à employer dépendent de la quantité de lait acide à manipuler. Puisque toute la chaleur nécessaire pour la séparation est utilisée pendant que le lait écrémé coagulé passe à tra vers l'éjecteur, c'est-à-dire pendant un temps très court, on économise du temps en multipliant le nombre des électeurs pour que la température désirée soit rapidement atteinte. Une valve précédant le tuyau du lait écrémé, conduisant de la cuve d'acidifieation à l'éjecteur, et une autre valve dans le tuyau de vapeur placé à côté de l'éjecteur, permettent un contrôle facile de la température à laquelle le lait écrémé doit être porté pour obtenir une séparation convenable de la caséine. La température nécessaire pour obtenir une séparation nette et rapide du caillé et du sérum dépend surtout de l'acidité initiale du lait écrémé, ce dont on peut s'assurer en prenant des échantillons de lait écrémé chauffé, alors qu'il sort de l'éjeeteur. Il reste à varier la température pour obtenir de meilleurs résultats au cas où l'essai effectué n'est pas satisfaisant.

Le caillé qui se rassemble dans la cuve inférieure forme chapeau et il laisse au-dessous le sérum clair.

Par suite de la rapidité de la séparation du caillé et du sérum, celui-ci peut être soutiré au travers de la valve grillagée, doublée d'un châssis de toile, qui se trouve à la partie inférieure de la seconde cuve. Il y a done continuité dans l'évacuation du sérum, ce qui permet de gagner du temps et d'utiliser une cuve plus petite.

Quand le sérum s'est écoulé et que la cuve est presque remplie par de la caséine, on recouvre celle-ci avec de l'eau, ee qui raffermit le grain et facilite le travail.

On peut procéder à plusieurs lavages du caillé obtenu sous une forme très granuleuse ; ils conduisent à une bonne caséine. 
Lorsque le dernier lavage est assuré, le caillé est mis à égoutter avant d'êtrè pressé. Il se travaille bien, et il n'est pas aussi dur à moudre que celui que l'on obtient par la méthode d'acidification par l'acide sulfurique ou l'acide chlorhy. drique.

mÉtHOde du SÉRUM ACIDE. - Elle consiste à précipiter la caséine du lait écrémé au moyen de l'acide lactique qui se trouve dans des sérums obtenus les jours précédents et d'une haute acidité.

Cette technique correspond à la seconde technique argentine décrite plus haut.

Le sérum est ajouté en quantité suffisante pour qu'on obtienne ane séparation continue du caillé quand on le mélange avec le lait écrémé, tel qu'il sort de l'écrémeuse.

Le lait écrémé et le sérum aeide, chauffés chacun de leur côté à une température de $54^{\circ}$ à $57^{\circ} \mathrm{C}$. $\left(130^{\circ}\right.$ à $150^{\circ} \mathrm{F}$.), sont amenés dans un tuyau commun possédant une petite auge, où ils se mélangent en proportions convenables pour que la précipitation de la caséine se produise, puis toute la masse est déchargée dans une eaisse dont le fond est constitué par un tamis recouvert d'une toile.

Le lait et le sérum sont portés à la température convenable par de la vapeur provenant d'un premier éjecteur posé sur le passage du lait écrémé, et d'un second, placé sur le passage du sérum provenant d'une cuve surélevée.

Du sérum est recueilli, puis pompé dans le bac où se trouve la réserve et où il va s'acidifier lui-même. Le caillé qui s'est accumulé dans la caisse au-dessus du tamis, est pressé de la manière habituelle ou laissé s'égoutter jusqu'au lendemain matin.

caséine a l'acide sulfurique. - Deux procédés peuvent être employés ici, celui du caillé dit pressé et celui du caillé cuit.

a) Procédé du caillé pressé. - Le lait frais écrémé est chauffé dans une cuve par de la vapeur directe vers $49^{\circ}$ C. $\left(120^{\circ} \mathrm{F}\right.$.), mais jamais plus haut que $52^{\circ}$ $\left(125^{\circ}\right.$ F.). Une solution d'acide sulfurique faite par le mélange d'un demi-litre d'acide commercial, de densité 1,83 et de 2 litres $1 / 4$ d'eau (le texte a méricain dit : une pinte, soit 01 . 57, et quarts d'eau, soit 21.28 ), est ajoutée à 450 litres de lait éerémé frais. Le caleul montre qu'il faut $1.048 \mathrm{gr}$. d'acide sulfurique pour 440 litres de lait écrémé.

On doit agiter d'une manière continue pendant l'addition de l'acide, jusqu'à ce qu'une bonne séparation du caillé soit obtenue, ce qui est indiqué par le sérum clair. Ceci ne demande que quelques minutes si une quantité suffisante d'acide a été utilisée.

Dans le cas où les flocons se séparent mal du sérum, on ajoute, avec beaucoup de précautions et graduellement, un peu plus d'acide, pour obtenir un résultat convenable, mais jamais plus qu'il n'est nécessaire ; on s'arrête lorsque la séparation nette est obtenue. La quantité d'acide varie, évidemment, avec la force de l'acide commercial vendu et l'état du lait écrémé.

Le caillé est rassemblé loin de la valve grillagée d'écoulement, pu celle-ci pour laisser passer le sérum. Ensuite, on refermela valve et on recouvre le 
caillé d'eau froide ; on lave en agitant bien. Après l'égouttage de l'eau de lavage, le caillé est pressé, ou traité selon le procédé qui suit :

b) Procédé du caillé cuit. - Ce procédé élimine le pressage du caillé et ne demande, pratiquement, comme équipement, que les. bacs de précipitation. Aussi peut-il être adopté quand la laiterie peut vendre le caillé humide à une usine centrale de dessiccation se trouvant à proximité.

D'après A, O. DAHLBerg, la caséine de caillé cuit donne une. solution d'une plus haute viscosité que la caséine d'autoacidification lactique, quand on la dissout dans certains solvants.

La précipitation se fait dans les mêmes conditions qu'avec le caillé pressé, c'est-à-dire que le lait écrémé est chauffé à $49^{\circ}-50^{\circ}$ (aux environs de $120^{\circ} \mathrm{F}$.) et que l'acide sulfurique, dilué au préalable, est additionné en quantité convenable pour obtenir une séparation très nette. Après égouttage du sérum, le caillé est recouvert. d'eau et le mélange est chauffé jusqu'à $77^{\circ}-79^{\circ} \mathrm{C}$. ( $170^{\circ}$ \&े $175^{\circ} \mathrm{F}$.), par action directe de la vapour. A cette température, tout le caillé se ramasse en une masse plastique semifluide. L'eau est entraînée et, après pétrissage du caillé très mou résultant, en vue d'enlever autant que possible l'eau qui reste, on le place dans un baril où il se ramasse en une masse plutôt compacte qui, après refroidissement, devient très ferme et imperméable. Elle peut être gardée dans cet état pendant un jour, même dans une atmosphère chaude.

Lo caillé de l'opération du jour, fraîchement euit, est placé sur le caillé de la. veille qui a déjà une consistance ferme. Les barils recouverts sont expédiés à une usine de dessiccation centrale, sans avoir à craindre que les caillés s'effritent. Le caillé cuit est très dur à moudre et exige des meules spécialement fortes.

On veille, dans ce procédé de fabrication, à n'employer que juste: la quantité d'acide nécessaire, ear un excès de ce dernier nuit au travail du caillé lors de la euisson. Si, en effet, il y a trop d'acide, le caillé cuit dans l'eau ne se rassemble pas en une masse plastique. caractéristique, mais se brise en fines particules.

Lorsque la quantité d'acide sulfurique utilisée est convenable, l'acidité du sérum vis-à-vis de la soude, en présence de la phénolphtaléine, doit être de $0,30 \%$ à $0,32 \%$. Si elle dépasse $0,34 \%$, on observe des incidents de fabrication de la nature de ceux qui viennent d'être relevés, e'est-à-dire un effritement de la masse des caillés, et non pas une agglomération de leur masse.

Toute la difficulté de ce procédé de fabrication réside done dans: le soin qu'il faut apporter à n'utiliser que la quantité d'acide nécessaire. Aussi, des essais peuvent-ils être faits au préalable.

Il va sans dire que si la laiterie qui prépare la caséine par ee: procédé, sèche elle-même sa caséine, il n'est pas nécessaire de mettre: celle-ci en baril. La masse plastique des caséines est mise à égouttersur un plancher propre et légèrement en pente, sous forme de bandes: assez plates. 
CASÉINE A L'ACIDE CHLORHYDRIQUE. - Comme avec l'acide sulfurique, deux procédés sont utilisés : celui du caillé pressé et celui du caillé cuit.

a) Procédé du caillé pressé. - Avec le procédé du caillé pressé, le lait, nous dit A. O. DAнLBERG, ne doit pas étre chauffé au préalable à plus de $49^{\circ} \mathrm{C}$. $\left(120^{\circ} \mathrm{F}\right.$.). Il est préférable de chaufter aux environs de $46^{\circ} \mathrm{C}$. ( $\left(115^{\circ} \mathrm{F}\right.$.). L'emploi de températures dépassant $49^{\circ} \mathrm{C}$. $\left(120^{\circ} \mathrm{F}\right.$.) donnerait une caséine moins bonne.

L'acide chlorhydrique commercial de $20^{\circ}$ à $23^{\circ} \mathrm{B}$. est graduellement ajouté, pendant qu'on agite, jusqu' à ce que la précipitation soit assurée. Il faut, approximativement, 2 litres $1 / 2$ d'acide que l'on dilue au préalable avec une égale quantité d'eau, pour 900 litres de lait écrémé. Il faut prendre soin de ne pas ajouter trop d'acide, car un excès donnerait un caillé mou, difficile à travailler.

La caséine-acide préparée avec l'acide chlorhydrique donne des grains moins fermes et à tendance à se coller sur la toile, lors du pressage. Pour éviter cette difficulté, le caillé, aussitôt après l'égouttage du sérum, est recouvert d'eau tiède dans laquelle on ajoute un peu d'acide sulfurique, environ 75 à 80 gr. pour chaque 500 litres de lait écrémé travaillé. Cette opération reǹd le caillé plus ferme et plus facile à presser.

b) Procédé du caillé cuit. - Ce procédé est semblable à celui du même nom, que nous avons étudié un peu plus haut en employant l'acide sulfurique. Comme ce dernier, il supprime la nécessité de presser.

La précipitation s'effectue dans les mêmes conditions qu'avec l'acide sulfurique. Une fois que le sérum a été éliminé, le caillé est couvert d'eau froide et chauffé directement avec de la vapeur à $80-85^{\circ} \mathrm{C}$. (1750-185 $\mathrm{F}$.) qui amène la fusion d̀ peu près complète du caillé en une masse semi-fluide, plastique, souple, $\vec{i}$ condition, bien entendu, qu'un excès d'acide n'ait pas été employé.

Lorsque l'eau a fini de s'égoutter, la masse plastique est pétrie, afin de lui enlever les dernières traces d'eau, et on la traite comme il a été dit pour le caillé à l'acide sulfurique.

caséINE de caillé granuleux (Grain curd casein). - Dans le but d'obtenir une caséine uniforme et d'excellente qualité, devant répondre aux besoins de l'aéronautique des Etats-Unis pendant la guerre, le Bureau of Dairy Industry étudia la possibilité de son obtention. Cette caséine, "grain curd casein ", comme disent les Américains, - ce que nous traduisons par : caséine de caillé granuleux -, est particulièrement recommandable, nous dit A. 0 . DAHLBERG, quand le sérum doit être employé pour la récupération du lactose.

Sa préparation se ramène essentiellement à ceci : on précipite à basse température, avec une acidité nettement définie, en employant de 
l'acide chlorhydrique, beaucoup plus dilué que celui qui est utilisédans les procédés antérieurement décrits.

Dans ces conditions, on obtient un précipité granuleux dont l'aspect physique permet un lavage rapide et profond avec une eau acidifiée dont l'acidité est la même que celle de la cuve, lorsque la précipitation de la caséine est terminée. On a trouvé, en effet, que lorsque l'acide chlorhydrique, d'une concentration à peu près normale ( $\mathrm{N} / 1=36 \mathrm{gr}$. 50 d'acide chlorhydrique au litre), était ajouté au lait écrémé frais, à une température ne dépassant pas $34^{\circ} \mathrm{C}$., dans des proportions telles que le sérum obtenu ait un $p \mathrm{H}$ apparent de 4,6, avec le rouge de méthyle comme indicateur, le précipité avait un caractère remarquablement granuleux et une élasticité qui permettait un lavage sérieux, sans avoir à craindre le tassement de la masse, lequel se constate le plus ordinairement.

Ce procédé nécessite, il est vrai, un équipement additionnel, sous la forme de tamis d'égouttage et de sacs d'égouttage assez nombreux ; mais la qualité de la caséine obtenue est excellente et, de plus, le pressage et le séchage peuvent s'effectuer aisément.

L'aspect granuleux du précipité est tel, qu'il est possible de travailler la caséine obtenue, à la centrifugeuse.

Zoller (25) a fait des essais dans des conditions commerciales et il estime que le travail de la caséine obtenue par le procédé du " grain curd " peut très bien s'effectuer par centrifugation daps les grandes usines et, dans ces conditions, fait-il remarquer, les opérations de précipitation en bac, de lavage, de pressage, peuvent être entièrement mécaniques, ce qui permet de gagner du temps et de conduire d'une façon très homogène la suite des opérations.

La technique de ce procédé exige l'emploi d'indicateurs colorés qu'il faut utiliser dans des conditions bien déterminées. Ces indicateurs assurent un contrôle de l'acidité à réaliser, tant pour la précipitation de la caséine que pour l'acidification de l'eau de lavage. L'indicateur coloré employé en l'espèce est le rouge de méthyle. La solution est préparée en dissolvant $0 \mathrm{gr} .2$ de rouge de méthyle pur dans $1.000 \mathrm{~cm}^{3}$ d'alcool à $95^{\circ}$. Cette solution donne une couleur rouge vif lorsque $V$ gouttes sont additionnées à $10 \mathrm{~cm}^{3}$ de la liqueur à essayer. On s'habitue très vite, d'ailleurs, à l'appréciation de la teinte convenable et, pour cela, on peut se procurer des solutions étalons, de $p \mathrm{H} 4,6,4,8$ et 5 , afin de faire les comparaisons nécessaires; mais, très rapidement, l'ouvrier est à même d'apprécier la teinte qu'il lui faut obtenir lors de la préparation de la caséine.

Précipitation de la caséine. - La température de précipitation est un facteur très important pour assurer le caractère granuleux

(25) Indication 4 de Sutermeister, p. 102. J. Ind. Eng. Chem., 1921, 13, 510. 
typique à la caséine obtenue, et rendre son lavage extrêmement facile.

Si le lait est tout à fait frais, on peut opérer à $34^{\circ} 50$ (940 F.) ; s'il était légèrement acide, il faudrait descendre à $34^{\circ} \mathrm{C}$. (93 $93^{\circ} \mathrm{F}$.) ; mais, dans aucun cas, il ne faut dépasser $35^{\circ} 5$ C. ( $96^{\circ}$ F.) ou bien, si l'on allait au-delà, on aurait des masses compactes au lieu d'avoir une consistance granuleuse du précipité.

L'acide chlorhydrique est dilué avec 8 jois son poids d'eau ; il est additionné lente. ment, de préférence en le distribuant sur la longueur du bac au moyen d'un auget en bois, dont la rigole possède un grand nombre de petits trous. Pendant que l'acide arrive, on agite rapidement, pour incorporer, d'une façon très homogène, l'acide à la masse.

Quand la coagulation débute, l'acide est ajouté plus lentement encore, mais on ne cesse pas d'agiter.

Quand l'addition est terminée, la séparation de la caséine se fait très vite et le sérum obtenu est clair. On laisse le caillé se reposer dans la cuve et l'on soutire la moitié du sérum qui se trouve au-dessus, au travers d'un sac.

La quantité de sérum qui reste est amenée à un point défini d'acidité ; pour cela, on y apporte une proportion assez faible de la solution chlorhydrique antérieurement employée, jusqu'à ce que $10 \mathrm{~cm}^{3}$ de sérum avec $\mathrm{V}$ gouttes de rouge de méthyle décèle un $p H$ apparent de 4,6 à 4,8; puis on agite avec soin, mais non brutalement, pour briser la masse du caillé.

Le eaillé granuleux obtenu est ferme au toucher et les grains, comprimés dans la main, ne doivent pas se coller les uns aux autres, ce qui arriverait si l'on employait davantage d'acide.

Si l'acide est en quantité insuffisante, on obtient un caillé mou et, dans un cas comme dans l'autre, le lavage du grain et l'égouttage sont rendus plus diffieiles.

Une précipitation à une température trop basse donne un caillé mou, tandis qu'une température trop élevée donne un caillé rugueux, se rassemblant en amas; l'une et l'autre de ces consistances ne sont pas désirables.

A. O. Dahlberg parle de l'emploi du lait pasteurisé dans la préparation de la caséine en grains. C'est là une technique que l'on ne connaît pas dans les pays européens. En tout cas, d'après les recherches faites au Bureau de la Laiterie du Ministère de l'Agriculture des Etats-Unis, pendant la guerre, avec la collaboration de ZoLLER, ce dernier a constaté que la pasteurisation avait un inconvénient qu'on pouvait habituellement supprimer en faisant la précipitation à une plus haute température.

Les données suivantes de ZoLLER sont une approximation dont il est possible de tenir compte :

Pasteurisation $63^{\circ}$ pendant 30 minutes

$63^{\circ}$

$63^{\circ}$

$80^{\circ}$

$100^{\circ}$

$\begin{array}{lrl}n & 60 & n \\ n & 100 & n \\ n & 60 & n \\ n & 100 & n\end{array}$

Température de précipitation

$40^{\circ}$

$45^{\circ}$

$50^{\circ}$

$50^{\circ}$

$52^{\circ}$ 
C'est à l'acide chlorhydrique que l'on a surtout recours, dans ce procédé. Si l'on emploie l'acide sulfurique, ll est nécessaire de précipiter à une température de $2^{\circ}$ plus élevée qu'avec l'acide chlorhydrique, afin de s'assurer un caillé granuleux d'une consistance convenable.

Lavage du caillé. - Le reste du sérum est soutiré en retenant dans la cuve, autant que possible, tout le caillé. Celui-ci est alors recouvert avec de l'eau chlorhydrique qui a été amonée à une acidité d'un $p H 4,8$ à 5 .

L'acidité de l'eau de lavage est assurée en faisant des essais qui consistent à ajouter l'acide ehlorhydrique à l'eau par petites quantités, et en faisant des prises de $10 \mathrm{~cm}^{3}$, à chacune desquelles on ajoute $\mathrm{V}$ gouttes de la solution de rouge de méthyle, puis en comparant la couleur obtenue aveo celle des solutions étalons. On arrive rapidement à connaître la quantité d'acide qu'il faut employer pour les fabrications ultérieures.

Ioi, comme dans les premiers temps de l'opération, il faut éviter de descendre au-dessous du $p H$ 4,6. L'emploi d'un excès d'acide dans les eaux de lavage aurait les mêmes inconvénients qu'un excès d'acide lors de la précipitation.

On lave soigneusement, en agitant, de façon à assurer la rupture des masses qui finiraient par se former quand les particules du caillé reposent au fond de la cuve.

Lorsque le lavage est assuré, on draine le tout et le caillé est ramené sur le châssis d'égouttage où l'on complète, ultérieurement, son lavage.

Pressage. Séchage. - Le pressage et le séchage de la caséine de "grain curd" demandent un pəu plus de soins que s'il s'agissait de la précipitation d'un caillé ordinaire, ce qui est dû̀ à ce que $1 a$ caséine obtonue ainsi cède facilement son humidité. La pression sera plus légère au commencement; avec la caséine de " grain curd ", on élimine davantage d'eau en un temps plus court.

Quant au séchage et à la mouture de la caséine, il n'y a rien de spécial. Retenons simplement qu'une telle caséine se sèche et se moud exceptionnellement bien, domnant un produit d'une bəlle couleur, d'une consistance élastique non cassante, comm 3 on l'observe assez souvent avec la caséine obtenue par la précipitation sulfurique ou chlorhydrique ordinaire.

CASÉ INE de bABEuRre. - Aux Etats-Unis, on a pensé également à préparer de la caséine avec le babeurre; mais la richesse de ce dernier en matière grasse et la grande variation dans l'aspect physique de la plupart des babeurres apportent des difficultés dans la fabrication d'une caséine de bonne qualité, ce qui en a limité la production; elle est généralement vendue à un prix inférieur et est employée pour l'alimentation des animaux et des volailles. Ici, sa richesse en graisse est un avantage et sa couleur foncée est sans conséquence.

Il est bien préférable, dès l'instant où l'on vise à l'alimentation des animaux, notamment à celle des volailles, de dessécher purement et simplement le babeurre, comme cela est fait aux Etats-Unis. 
On ne peut pas songer à préparer de la caséine en partant du babeurre riche en eau et qui provient du barattage d'une erème très acide et préalablement pasteurisée. On peut utiliser soit du babeurre de crème douce, soit du babeurre de erème acide. Aux Etats-Unis, on baratte la crème douce, sans acidité acquise appréciable, en vue d'obtenir un beurre qui puisse se conserver pour être, plus tard, réémulsionné après fusion avee un lait écrémé obtenu en partant de la poudre de lait maigro.

A. O. Dahliberg reconnaît que la méthode de l'éjecteur est celle qui est particulièrement bien adaptée pour la fabrication de la caséine de babeurre de crème douce. Celle-ci donne un coagulum fin, granuleux, qui peut être aisément et facilement lavé et débarrassé des matières minérales, si bien que la caséine résultante a un taux faible de cendres.

Il est bon de passer le babeurre de crème douce à l'écrémeuse, afin de le débarrasser de sa matière grasse.

On pəut égalemənt préparar de la caséine avec du babeurre de crème dovce, en le laissant s'acidifier spontanément au repos, jusqu'è ce qu'un caillé ferme, mais cependant doux au toucher, se produise. Il n'est pas recommandé d'agiter exagérémont le caillé avant le chauffage, parce qu'on obtient des flocons trop fins et il en résulte des pertes. La température de chauffage doit osciller entre $46^{\circ}$ et $52^{\circ} \mathrm{C}$. $\left(115^{\circ}\right.$ à $\left.125^{\circ} \mathrm{F}.\right)$. Lo lavage avec de l'eau froide raffermit le grain, ce qui facilite les manipulations et améliore, en même temps, la qualité du produit. Fn raison de la richesse en matière grasse de cette caséine, elle ne doit pas ôtre desséchée à plus de $55^{\circ} \mathrm{C}$. (130 $\mathrm{F}$.).

\section{PRÉPARATION DE LA GASÉINE-PRÉSURE DANS L'INDUSTRIE}

\section{TECHNIQUE FRANĢAISE. - (DORNIC et CHOLLET.)}

"La cuve étant pleine de lait, on réchauffe celui-ci vers $35^{\circ}$ en mettant l'agitateur en marche ; lorsque cette température est atteinte on ajoute, s'il y a lieu, le chlorure de calcium et une quantité de présure telle que la coagulation ait lieu au moins 20 minutes après. Cette quantité dépend de la force de la présure, de l'acidité du lait et de l'addition ou non de chlorure de ealeium.

Il est souvent commode avee les présures concentrées, pour obtenir une répartition plus rapide dans la masse, de diluer cette présure avee une certaine quantité d'eau. Après avoir laissé l'agitateur faire un ou deux tours pour bien mélanger la présure au lait, on l'arrête et on attend que le lait soit coagulé; on reconnait le plus souvent qu'il en est ainsi en plongeant le doigt dans la cuve ; on peut saisir le moment précis où commence la coagulation en laissant le lait s'écouler goutte à goutte par la bonde placée à la base de la cuve; cet écoulement cesse dès que commence la coagulation. On met alors en marche l'agitateur, tournant à 130 tours à la minute, pour briser le caillé, et on ouvre la vanne de vapeur de façon à le "cuire "; on chauffe jusqu'à 60-63\%. L'opération doit durer une demi-heure environ et l'élévation de température doit être régulière, c'est-à-dire qu'on doit chercher à ce qu'elle s'élève d'environ un degré par minute. Quand le caillé est cuit, on ferme la vanne de vapeur et on place sous la bonde de la cuve un tamis métallique, ou bien on se contente d'y fixer un sac, de façon à retenir les grains de 
caillé qui pourraient être entraînés par le sérum. On ouvre la bonde et on laisse couler ce sérum.

Ceci fait, on ferme la bonde et on remplit la cuve d'eau, pour débarrasser la caséine des matières étrangères solubles qu'elle renferme, notamment du lactose ; sans cela, ce dernier brunit au séchage et déprécie beaucoup la marchandise qui ne paut alors servir à fabriquer que des matières plastiques opaques et colorées. On fait tourner l'agitateur pendant une demi-heure au moins, on évacue l'eau et on fait un second lavage dans les mêmes conditions. Lorsqu'on le peut, on effectue le premier lavage avee de l'eau tiède, à $25-30^{\circ}$.

La caillé est alors mis dans le pressoir, à l'aide d'une pelle; si on a une presse ordinaire, on le met dans des saes qui sont portés sous cette presse. On serre la vis. du pressoir deux ou trois fois dans la journée et on abandonne jusqu'au lendemain. A ce moment, on a la a caillebotte " qui contient 50 à $55 \%$ d'eau si elle a été conveblemənt pressée. Cətte caillebotte est brisée dans un moulin spécial - le moulin à caillé - et étendue sur des clisses en toile qui sont introduites dans le séchoir soit directoment (séchoir à tiroir), soit après avoir été disposées sur les chariots (séchoir à tunnel). Les chariots utilisés dans les tunnels peuvent porter 80 clisses chacun.

Toutes choses égales d'ailleurs, le séchage est d'autant plus rapide qu'on met moins de caillebotte sur chaque elisse; les clisses de $1 \mathrm{~m} .10 \times 0 \mathrm{~m} .60$ peuvent recevoir de 2 à $3 \mathrm{~kg}$. de caillebotte. Le courant d'air obtenu, en envoyant à l'aide du ventilateur l'air sur les batteries de radiateurs chauffés, ne doit pas avoir une température supérieure à $50-55^{\circ}$; dans ces conditions, le séchage dure 6 à 7 heures environ ; au bout de 3 ou 4 heures, si on veut avoir un séchage bien uniforme, on chang teurs soient à l'autre extrémité, et inversement. Dans les séchoirs à tiroirs, on ehanga les elisses de place dans les mêmes conditions. Un courant d'air tropchaud (au-dessus de 56-60 C.) fait jaunir la caséine. Le même inconvénient se produit d'ailleurs lorsque, la easéine étant sèche, on la laisse dans le séchoir incomplètemant refroidi, par exemple quand le séchage étant terminé tard dans la soirée, on attend le lendemain pour enlever les clisses. La caséine sèche est passée dans un moulin à caillé, mise en sacs, et conservée dans un endroit sec ; sa teneur en eau est de 9 à $12 \%$. Une caséine qui renferme plus de $12 \%$ d'eau se conserve mal et ne paut être réduite en farine ; aussi le commerce de la caséine exige-t-il, en général, que ce taux ne soit pas dépassé. Pratiquement, une caséine bien sèche craque sous la dent, alors qu'une easéine encore humide s'écrase. facilement, $x$

Dans certains procédés nouveaux (BrLlrez), la caséine, lors de sa dessiccation, est déplacée lentement et automatiquement. Il y a ainsi un renouvellement continu des surfaces et l'on peut se contenter d'une température moindre. La dessiccation s'opère donc ici à une température plus basse et exige moins de temps.

\section{TECHNIQUE ARGENTINE.}

On recommande, en Argentine, d'employer du lait tout à fait. frais, écrémé très soigneusement. Le lait dont l'acidité est notable est réservé pour la fabrication de la caséine-acide. 
L'addition de la présure se fait lorsque le lait est à $35^{\circ}-37^{\circ} \mathrm{C}$, température un peu plus élevée que celle à laquelle on a recours dans la région des Charentes. Lorsque la coagulation commence (elle ne doit pas se faire avant 30 minutes), on agite, pour que la caillebotte reste en grains et ne se prenne pas en masse. Au début, l'agitation doit être vigoureuse. Une fois que le grain est de la grosseur d'un grain de riz, on ralentit l'agitation. Si celle-ci est trop énergique, le caillé reste en poussière ; on obtient un mauvais rendement. $\Pi$ y a là un tour de main qu'un ouvrier attentif aequiert rapidement. Le brassage doit durer au moins 45 minutes et, pendant qu'il a lieu, on élève graduellement la température, de façon à atteindre $70^{\circ} \mathrm{au}$ moins. L'élévation de température n'est done pas tout à fait de un degré par minute. De $37^{\circ}$ à $50^{\circ}$, le chauffage est lent. Il s'accentue lorsqu'on passe de $55^{\circ}$ à $70^{\circ}$. A l'intérieur de la cuve, on place une planchette sur le pourtour de laquelle la eaillebotte vient se briser. Cela empêche que toute la masse soit entraînée dans un mouvement régulier et de même sens.

Lorsque ces opérations sont terminées, on arrête l'agitation et on évacue rapidement le sérum, en le filtrant sur toile ou sur un tamis métallique serré, afin d'éviter les pertes qui se produiraient par entraînement. Quand le sérum est éliminé, on ajoute de l'eau froide. On agite mécaniquement pour laver le grain, en même temps que pour rafraîchir et raffermir ce dernier.

On conseille d'additionner l'eau de lavage de $1 \mathrm{~cm}^{3}$ de formol par hectolitre de lait écrémé travaillé. Lorsque le temps est orageux, on peut doubler ou tripler cette dose et, dans ce cas, on ne le fera que pour le deuxième lavage. La quantité doit en être fixée très exactement car, s'il y avait un excès de formol, la caséine obtenue serait difficilement employable pour les usages auxquels elle est ordinairement destinée.

La caillebotte est mise dans des sacs, transportée à l'usine de séchage - quand elle n'est pas desséchée à l'usine même -, où elle est pressée très vigoureusement, puis passée du moulin à caillé au séchoir.

\section{TECHNIQUE AMERICAINE.}

Le lait écrémé est porté à une température de $35^{\circ} 5 \mathrm{C}$. ( $\left(96^{\circ} \mathrm{F}\right.$.) et additionné d'une quantité suffisante de présure préalablement diluée avec de l'eau, pour obtenir la coagulation en 15 à 20 minutes. Un essai préliminaire fixe la quantité de présure à utiliser.

Le moment du rompage du caillé et son chauffage sont importants à fixer et devront être modifiés selon les eirconstances.

Une bonne règle à suivre, dit $\mathrm{A}$. O. DAHLBERG, est de noter le temps qui s'écoule depuis l'addition de la présure jusqu'au premier signe de la coagulation, et d'ajouter approximativement au temps observé son dixième environ ; aussitôt après, on rompt le caillé. Le chauffage du caillé, soit par le moyen d'une cuve à. double fond, soit par la vapeur admise directement dans la cuve, est commencé dès que le rompage débute et il est continué jusqu'à ce que la température atteigne $65^{\circ} 5$ ( $150^{\circ}$ F.). Certains techniciens recommandent d'agiter continuellement le lait écrémé pendant la coagulation et de chauffer à une température plus élevée que celle indiquée ci-dessus. Cette température atteinte, on laisse le caillé au repos pendant 10 minutes. Le sérum clair est soutiré aussi rapidement que possible à travers une valve grillagée, puis, quand il est évacté, on ferme la valve et l'on. recouvre le eaillé, immódiatement, avec de l'eau tiède aux environs de $25^{\circ}$. Le: 
mélange est agité pəndant quelques minutes, en prenant soin de détacher le caillé du fond de la cuve ; on laisse reposer à nouveau. On évacue cette première eau, on recommence le lavage une fois et même deux foik, toujours avec de l'eau viède, d'une température un pəu plus élevée, pour que la masse du caillé soit entre $26^{\circ} 6$ et $32^{\circ} 3$ C. $\left(80^{\circ}\right.$ et $90^{\circ}$ F.). Une température plus basse aurait tendance à donner un produit dur quand il est sec. L'évacuation de la dernière eau de lavage a lieu en même temps que celle du caillé, qui est reçu sur un tamis d'égouttage. Un soin tout particulier est apporté au pressage et au séchage. La eaillebotte abandonne assez facilement son humidité ; elle est pressée fortement pendant une heure, passée av moulin à broyer, étalée sur les clisses. La dessiccation doit avoir lieu à $43^{\circ} 3-46^{\circ}\left(110^{\circ}\right.$ à $115^{\circ} \mathrm{F}$.). Une plus haute température ou un séchage plus lent donnent une couleur jaune, indésirable.

La fabrication de la caséine-présure demande plus de soins que celle de la easéine-lactique, mais, quand le lait écrémé est très frais et que l'on prend toutes les précautions qui viennent d'être indiquées, aucune difficulté réelle ne peut être rencontrée pour obtenir un produit satisfaisant.

DONNEES ANAL YTIQUES SUR LES CASÉINES COMMERCIALES.

Nous rassemblons ici dans un même sous-chapitre les données assez récentes que nous avons empruntées à différents auteurs, tant sur la caséine-présure que sur la caséine-acide.

Les tableaux suivants ont déjà paru dans un travail de Ch. Porcher et Mile J. Brigando (26).

TABLEAU I (27).

\begin{tabular}{|c|c|c|c|}
\hline & Eau $\%$ & Graisse & Cendres \\
\hline \multirow{5}{*}{ Caséine-présure. } & 10,62 & 3,0 & $5,4 \quad(a)$ \\
\hline & 11,90 & 3,4 & $6,5 \quad(a)$ \\
\hline & 11,34 & 1,83 & $6,10(a)$ \\
\hline & 12,02 & 1,0 & $6,56(a)$ \\
\hline & 8,76 & 1,50 & 7,76 \\
\hline \multirow{4}{*}{ Caséine-lactique } & 9,52 & 3,0 & 2,20 \\
\hline & 10,06 & 4,2 & 2,60 \\
\hline & 6,46 & 1,25 & 2,56 \\
\hline & 10,12 & 2,25 & 2,91 \\
\hline
\end{tabular}

(26) Les différences analytiques de la caséine-acide et de la caséine-présure. Ann. des Falsif. et des Fraudes, 1929, 22, 143.

(27) Dornic et M. DAIRE. Revue Générale du Lait, 1908-1909, 7, 328.

(a) Ces caséines ne sont pas assez chargées en matières minérales ; sans doute ont-elles été préparées avec des laits déjì acides. 
Tableau II (28).

GASÉINES-AGIDES.

\begin{tabular}{|c|c|c|c|}
\hline & Eau & Graisse & Cendres \\
\hline Moyenne ............. & 6,67 & 0,06 & $0,08(a)$ \\
\hline Minimum ............ & 1,65 & 0,01 & 0,00 \\
\hline Maximum...$\ldots \ldots \ldots$ & 9,62 & 0,09 & 0,49 \\
\hline \multicolumn{4}{|c|}{ Caséines-présure } \\
\hline Moyenne .............. & 3,84 & 0,20 & 7,07 \\
\hline Minimum ............ & 0,60 & 0,08 & 5,00 \\
\hline Maximum ............ & 10,85 & 0,55 & 8,55 \\
\hline
\end{tabular}

(28) Tableau emprunté à H. Droop Rrchmond. Dairy Chemistry, $3^{e}$ éd., 1920.

(a) Il s'agit certainement ici de caséines préparées au laboratoire.

TABLeaU III.

ANALYSES DE GASEINES “ GRAIN-GURD 》 (29).

(Fabriquées dans de grandes usines sur le type commercial.)

\begin{tabular}{|c|c|c|c|}
\hline Usines & Humidité \% & Cendres $\%$ & Graisse $\%$ \\
\hline $\mathrm{A}, \ldots \ldots \ldots, \ldots$ & 7,37 & 2,55 & 0,15 \\
\hline B $\ldots \ldots \ldots \ldots \ldots \ldots$ & 7,54 & 2,78 & 0,24 \\
\hline $\mathrm{C} \ldots \ldots \ldots \ldots \ldots$ & 7,41 & 2,86 & 0,21 \\
\hline $\mathrm{D} \ldots \ldots \ldots \ldots \ldots \ldots$ & 7,13 & 2,90 & 0,08 \\
\hline $\mathrm{E} \ldots \ldots \ldots \ldots \ldots \ldots$ & 8,34 & 1,90 & 0,11 \\
\hline$F \ldots \ldots \ldots \ldots \ldots$ & 8,73 & 2,10 & 0,11 \\
\hline
\end{tabular}

(29) Ce tableau et les deux suivants sont empruntés au livre de TAGUE : CLARK, ZOLLER, Dahlberg et Wetmar. Journ. Ind. and Eng. Chem., 1920, 1, 12, 1163. 


\section{Tablead IV.}

ANALYSES DE GASÉINES TYPIQUES (29):

\begin{tabular}{|c|c|c|c|}
\hline Types & Humidité \% & Cendres $\%$ & Graisse $\%$ \\
\hline Caillé cuit ........... & 8,56 & 6,03 & 0,08 \\
\hline Caillé sulfurique ....... & 8,00 & 4,12 & 0,33 \\
\hline Lait auto-acidifié ...... & 7,14 & 3,45 & 0,24 \\
\hline "Grain-Curd" ....... & 7,41 & 2,96 & 0,21 \\
\hline
\end{tabular}

\section{Tableau $V$.}

ANALYSES DE GASÉINES TYPE " GRAIN-GURD ".

(Préparées au laboratoire sur une échelle semi-commerciale (29).

\begin{tabular}{|c|c|c|}
\hline Condition du lait & Humidité $\%$ & Cendres $\%$ \\
\hline Vieux lait écrémé ............ & 7,70 & 1,43 \\
\hline Lait écrémé acide $\quad \ldots \ldots \ldots \ldots \ldots \ldots \ldots$ & 6,25 & 1,36 \\
\hline Lait écrémé frais . ................. & 5,52 & 1,55 \\
\hline Lait écrémé frais $\ldots \ldots \ldots \ldots \ldots \ldots \ldots$ & 7,93 & 1,90 \\
\hline Lait fortement acide $\ldots \ldots \ldots \ldots \ldots \ldots$ & 7,32 & 1,79 \\
\hline 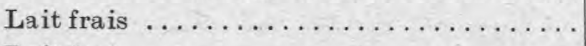 & 5,43 & 1,83 \\
\hline Lait frais $\ldots \ldots \ldots \ldots \ldots \ldots \ldots \ldots \ldots$ & 7,44 & 1,57 \\
\hline 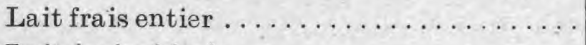 & 8,94 & 1,85 \\
\hline 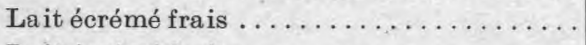 & 5,03 & 2,03 \\
\hline 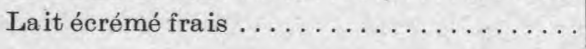 & 5,81 & 2,03 \\
\hline
\end{tabular}

Tableau VI.

DIVERSES CASÉINES (30).

\begin{tabular}{r|c}
\hline \hline & Cendres \% \\
Caséine "Grain Curd $\ldots \ldots \ldots \ldots \ldots$ & 1,8 \\
Caséine lactique $\ldots \ldots \ldots \ldots \ldots \ldots$ & 2,5 \\
Caséine précipitée par des acides & \\
minéraux $\ldots \ldots \ldots \ldots \ldots \ldots \ldots$ & 4,0 \\
Caséine-présure $\ldots \ldots \ldots \ldots \ldots \ldots$ & 8,6 \\
\hline \hline
\end{tabular}

(30) Rahn et Sharp, Physil der Milchwirtschaft, p. 193. 
Tableau VII.

CASÉINES DIVERSES (30 et 31 ).

\begin{tabular}{|c|c|c|c|}
\hline & \multicolumn{3}{|c|}{ Cendres } \\
\hline & Maximum & Minimum & Moyenne \\
\hline Etats-Unis. & & & \\
\hline Grain Curd $\ldots \ldots \ldots \ldots \ldots \ldots$ & 2,87 & 2,53 & 2,78 \\
\hline Babeurre .................... & $\sqrt{1}+2$ & & 3,28 \\
\hline Laetique .................... & 5,38 & 2,08 & 3,46 \\
\hline Sulfurique $\ldots \ldots \ldots \ldots \ldots \ldots \ldots$ & 5,34 & 2,62 & 3,92 \\
\hline Chlorhydrique ................ & 6,10 & 340 & 4,52 \\
\hline Lab.......................... & 8,39 & 7,18 & 7,97 \\
\hline 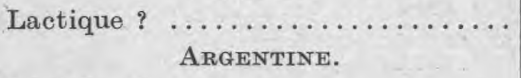 & 5,59 & 3,08 & 4,43 \\
\hline $\begin{array}{c}\text { Lactique } \quad \ldots \ldots \ldots \ldots \ldots \ldots \ldots \\
\text { Etats-Unis. } \\
\text { Caséines préparées spécialement. }\end{array}$ & 4,30 & 3,62 & 3,87 \\
\hline 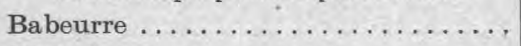 & 2,18 & 0,88 & 1,36 \\
\hline 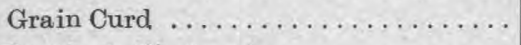 & 3,76 & 1,08 & 1,65 \\
\hline Lactique (éjecteur) .............. & 2,62 & 1,33 & 2,15 \\
\hline Sulfurique (éjecteur) . . . . . . . . & 5,36 & 3,02 & 4,05 \\
\hline Sulfurique lavée à chaud . ........... & 5,58 & 3,92 & 4,25 \\
\hline HCl lavée à chaud................ & 5,09 & 4,14 & 4,71 \\
\hline HCl lavée à chaud................ & 6,05 & 5,51 & 5,74 \\
\hline
\end{tabular}

(30) RAHN et ShaRP, p. 192.

(31) Browne, Journ. Ind. and Eng. Chem., 1919, 13, 119.

Tableau VIII.

ANALYSES GOMPARÉES DE GASÉINES DE LAIT ÉGRÉMÉ ET DE BABEURRE (32).

\begin{tabular}{|c|c|c|c|}
\hline Origine & Humidité $\%$ & Graisse \% & Cendres $\%$ \\
\hline Lait écrémé indigène . . . . . . . . . . & 7,80 & 0,60 & 2,80 \\
\hline Lait écrémé indigène ............ & 8,28 & 0,93 & 2,44 \\
\hline Lait écrémé étranger $\ldots \ldots \ldots \ldots \ldots$ & 7,95 & 0,66 & 1,89 \\
\hline Lait écrémé indigène $\ldots \ldots \ldots \ldots \ldots \ldots$ & 6,44 & 1,04 & 3,69 \\
\hline Lait écrémé indigène ............ & 7,74 & 0,93 & 1,14 \\
\hline Lait écrémé français $\ldots \ldots \ldots \ldots \ldots$ & 8,43 & 0,75 & 1,86 \\
\hline Babeurre..$\ldots \ldots \ldots \ldots \ldots \ldots \ldots$ & 7,65 & 6,54 & 0,99 \\
\hline Babeurre ................... & 6,68 & 5,00 & 1,30 \\
\hline Babeurre $\ldots \ldots \ldots \ldots \ldots \ldots \ldots$ & 6,82 & 9,52 & 0,60 \\
\hline Babeurre $\ldots \ldots \ldots \ldots \ldots \ldots \ldots \ldots$ & 5,86 & 5,62 & 0,76 \\
\hline Babeurre.$\ldots \ldots \ldots \ldots \ldots \ldots \ldots$ & 6,42 & 5,82 & 1,19 \\
\hline
\end{tabular}

(32) Dahlberg. U. S. Dept. of Agric., Bulletin 661, 1918. 


\section{Tablead IX.}

ANALYSES GOMPARÉES DE CASÉINES ACIDES ET DE CASÉINES PRÉSURE $(33,34,35)$.

\begin{tabular}{|c|c|c|c|c|}
\hline Type de caséine & $\underset{\%}{\text { Humidité }}$ & $\begin{array}{c}\text { Cendres } \\
\%\end{array}$ & $\begin{array}{c}\text { Graisse } \\
\%\end{array}$ & Auteur \\
\hline x & & & & \\
\hline Caséine A. I, . ..... & $7,27-10,50$ & $3,53-4,95$ & $0,23-1,71$ & Hopfner et \\
\hline & Moy. : 9,23 & Moy. : 4,07 & Moy. : 0,85 & Burmeister (34) \\
\hline Caséine-acide $\quad \ldots \ldots \ldots$ & $6,0-10,0$ & $2,2-2,9$ & $1,0-4,2$ & Kühl \\
\hline Caséine-acide ............ & $1,65-9,62$ & $0,0-0,5$ & $0,005-3,13$ & Burr (35) \\
\hline Caséine-présure .......... & $8,5-12,0$ & $5,4-7,8$ & $1,0-3,4$ & Kühl \\
\hline Caséine-présure . . . . . . . & $0,6-10,85$ & $5,0-8,55$ & $0,8-0,55$ & Burr \\
\hline
\end{tabular}

(33) Zoller. Journ. Ind. and Eng. Ohem., 1920, 1, 12, 1171.

(34) Hoptener et Burmeister. Chem. Ztg., 1912, 36, 1053.

(35) BURr. Milchwirtsch. Zeit., 6, 386.

\section{QUELQUES REMARQUES}

\section{AU SUJET DES TABLEAUX PRÉCF́DFNTS.}

Si nous jetons un coup d'œi'? d'ensemble sur les modes de préparation de la easéine dont il rient d'être question, nous voyons qu'ils se distinguent les uns des autres par des différences quelquefois légères, d'autres fois plus marquées, et les caséines qui en dérivent ne sont point semblables par leur aspect (couleur, consistance du grain, etc.), par leur richesse en matières minérales, par la viscosité de leurs solutions alcalines. Peut-on se baser, comme l'ont voulu certains auteurs, sur les différences dans les techniques et l'aspect des produits en résultant, pour avancer que ceux-ci n'ont pas tout à fait la même constitution ? Certainement non. Entre les procédés du laboratoire, plus sévères, plus minutieux, ceux de : Hammarsten, de L. L. van Slykn et J. C. BAker, de Ch. Porchirr, ete, et ceux de l'industrie, qui sont fort loin d'être aussi rigoureux, il y a, évidemment, des degrés. Notre intention est de nous employer à rechercher la raison de la variabilité des chiffres trouvés à l'analyse.

Si les caséines diffèrent entre elles, cela est dû à la technique à laquelle on a recours, aux soins qu'on apporte dans son exécution, puisque, en partant d'un même lait, on peut obtenir des produits industriellement différents.

Il nous reste donc à comprendre pourquoi, avec une substance chimique bien déterminée : la caséine, théoriquement toujours semblable à elle-même, on peut obtenir toute la variété des produits que l'industrie jette sur le marché. Ce qu'il faut incriminer ici, ce n'est pas vraiment la substance telle qu'elle se trouve originellement dans le lait, mais les circonstances qui entourent le processus 
d'acidification que nous mettons en œuvre pour obtenir le produit industriel qui en dérive, circonstances qui se ramènent essentiellement à l'emploi ou non de la chaleur, au brassage, à la compression du caillé, à la cuisson de celui-ci.

Certaines caséines-acides jetées sur le marché sont fortement acides, ce qui nuit à leur valeur marchande. Nous avons remarqué que les caséines les plus acides sont souvent aussi les plus minéralisées, ce qui s'explique par le manque de soins dans leur préparation, par l'insuffisance des lavages notamment.

La masse de caséine d'acidification lactique spontanée peut être considérée comme une éponge dans les mailles de laquelle se rassemble le sérum du lait. Une telle image peut s'appliquer également aux gros flocons d'acidification provoquée, lorsque cette acidification est effectuée brutalement. Ce n'est là qu'une explication mécanique qui ne peut toutefois nous donner entière satisfaction pour nous permettre de comprendre que la protéine soit fortement minéralisée, alors que théoriquement elle devrait être débarrassée de toute charge saline. Nous devons recourir à d'autres conceptions.

La caséine, quand elle vient d'être préparée, est très chargée d'eau. Lorsqu'on la met au séchoir, si le lavage a été insuffisant, l'eau s'évapore et laisse dans la trame de la protéine les sels qu'elle tient en solution.

On peut aussi faire intervenir les forces attractives dont les surfaces sont le siège, comme c'est le cas toutes les fois qu'on a à faire à des particules dispersées. C'est au jeu de ces forces que répond l'idée d'adsorption, laquelle se traduit par la fixation, à la surface des particules, des sels appartenant au milieu dans lequei elles sont dispersées.

Nous pourons aussi invoquer le phénomène de Donnan. Il nous est possible d'assimiler la surface des flocons de caséine à une membrane séparant deux solutions salines, le sérum, d'une part, et le liquide qui gonfle les flocons, d'autre part. L'équilibre de DONNAN exige que la solution saline qui se trouve dans la caséine gonflée, soit plus concentrée en cations que celle de l'extérieur.

Une indication importante est à retenir : c'est la nécessité d'atteindre le point isoélectrique. La chaux, la magnésie, qui se trouvaient à l'état colloïdal dans le complexe, unies, partie à la caséine et partie ¿ l'acide phosphorique, sont, au point isoélectrique, entièrement dissoutes.

L. L. VAN SLYKe et J. C. BATER ont noté ce fait important: lorsque les phosphates insolubles calciques et magnésiens ne sont pas complètement dissous, avant la précipitation de la caséine, il n'apparaît plus possible de les enlever en totalité après la précipitation, tellement ils sont fixés sur la trame protéique. 
Les cendres des caséines-acides sont constituées surtout d'acide phosphorique et de chaux. L'acide phosphorique provient, pour une partie, des phosphates calciques fixés sur la trame protéique et, pour le reste, du phosphore de la caséine.

Dans le tableau II, ainsi que nous le disons en note, le faible chiffre des cendres nous montre qu'il s'agit certainement ici de caséine préparée au laboratoire. Nous aurons l'occasion de montrer, au sujet de nos propres recherches, que l'industrie pourrait viser à obtenir des chiffres très bas, de l'ordre de celui qui est indiqué dans le susdit tableau, comme maximum par Droop RICHMond : 0,49 .

Dans le tableau $V$, qui donne des analyses de caséine du type "grain curd", nous voyons que le pourcentage des cendres est de l'ordre de 1,4 à 2,0\%. Ce sont, en général, les laits très acides qui donnent les plus faibles pourcentages.

Les caséines dont le caillé est cuit ont un chiffre de cendres élevé, qu'il s'agisse de caséine-chlorhydrique ou de caséine-sulfurique (tableau VII).

Nous pourrions déjà rapprocher l'examen de quelques-unes des circonstances dans lesquelles les caséines ont été obtenues, - circonstances que nous avons notées en italique dans les modes de préparation relevés plus haut - de l'analyse de leurs cendres, et chercher à établir une relation entre le taux de celles-ci et telle ou telle des circonstances relevées par nous. Nous croyons qu'il est préférable de ne tenter cette critique des procédés de préparation déjà examinés, qu'après l'exposé de nos propres recherches.

\title{
LE LIQUIDE DANS LE BEURRE
}

\author{
par \\ B. VAN DER BURG \\ Professeur à l'Ecole d'Agriculture de Wageningen (Pays-Bas).
}

(Fin.)

Pour le moment, ce qui est intéressant, c'est que si nous admettons la théorie de RAHN, ou si nous nous tenons à la conception plus simple de la formation du beurre que nous venons de mentionner, nous devons admettre dans les deux cas que les granules de beurre se sont constitués par la réunion des globules de matière grasse, et non par la confluence de la matière grasse en une phase continue, dans laquelle le sérum de lait est réparti pour faire en quelque sorte une émulsion. Nous basant sur notre conception, nous pouvons 